\title{
Micellar Formation in Aqueous Milieu from Biodegradable Triblock Copolymer Polylactide/Poly(ethylene glycol)/Polylactide
}

\author{
Li LIU, ${ }^{\dagger}$ Chenxi LI, Xiaohang LIU, and Binglin HE \\ The State Key Laboratory of Functional Polymer Materials for Adsorption and \\ Separation, Institute of Polymer Chemistry, Nankai University, \\ Tianjin 300071, People's Republic of China
}

(Received February 1, 1999)

\begin{abstract}
The formation of polylactide/poly(ethylene glycol)/polylactide (PLA/PEG/PLA) triblock copolymer micelles in aqueous milieu was studied by a combination of fluorescent probe, dynamic light scattering (DLS) and transmission electron microscopy (TEM) techniques. The fluorescence spectroscopy measurements showed that the PLA/PEG/PLA triblock copolymers were associated to form core-shell type polymeric micelles in water and the critical micelle concentration (CMC) values changed relatively little with increasing PEG chain length in the block copolymers. From dynamic light scattering investigations, the size of polymeric micelles was influenced by the molecular weights and the composition of two components of the triblock copolymers. The size of micelles increased with increasing PLA chain length in the block copolymer. Transmission electron microscopy observations revealed the formation of spherical micelles. The triblock copolymer underwent degradation after 13 days of hydrolysis in phosphate buffer solution (PBS). The degradation influenced the size distribution and the morphology of the micelles.

KEY WORDS Micelle / Biodegradable / Triblock Copolymer / Polylactide / Poly(ethylene glycol) /
\end{abstract}

Recently, biodegradable amphiphilic block copolymers have become attractive in both fundamental and applied fields of polymer science. ${ }^{1-4}$ Amphiphilic block copolymers are assembled in aqueous media to form polymer micelles with a core-shell structure, ${ }^{5}$ the hydrophobic core surrounded by hydrophilic corona. The formation of a corona of hydrophilic segments surrounding the core of water-incompatible segments prevent the progressive aggregation of the core and stabilize the micelles of amphiphilic block copolymers in an aqueous milieu. Block copolymer micelles are characterized by their size (a few tens to a few hundred of nanometers in diameter) and stability (low critical association concentration). ${ }^{6-8}$ Because of the existence of hydrophobic core, micelles may act as a microreservoir for various substances. These unique characteristics offer block copolymer micelles a special advantage for use in various applications including separation technologies ${ }^{9}$ and drug delivery systems, etc. ${ }^{2,4,10,11}$

During the last two decades, polymeric micelles based on biodegradable amphiphilic block copolymers have shown promise as drug carries for parenteral delivery systems. Recently, block copolymers prepared from PEG and lactides or PEG and lactic acid received considerable attention in view of applications in drug delivery systems. ${ }^{1,12,13}$ Poly(ethylene glycol) (PEG) has outstanding physico-chemical and biological properties, including hydrophilicity, solubility in water and in organic solvents, lack of toxicity, ${ }^{14}$ and absence of antigenicity and immunogenicity, ${ }^{15}$ which allowed PEG to be used for many biomedical and biotechnological applications. The copolymerization of PEG and lactides offered the possibility of varying hydrophilic/hydrophobic and soft/hard segment ratios and, thus, constitutes a very attractive means to modulate the basic properties of each homopolymer. So far, the synthesis and charac-

\footnotetext{
$\dagger^{\dagger}$ To whom all correspondence should be addressed.
}

terization of polylactide/poly(ethylene glycol)/polylactide (PLA/PEG/PLA) triblock copolymer have been carried out by many research groups. ${ }^{16-18}$ However, little attention was paid on the investigation of the micellar formation of this triblock copolymer and the degradation of the micelles in buffer solution. In water, a PLA/ PEG/PLA triblock copolymer chain can form a loop (both PLA blocks belong to the same micellar core), a bridged (each PLA block is in a different core), or a dangling chain. The formation of bridged micelles is one feature distinguishing this triblock copolymer from self-associated diblock copolymer. It is noted that the concentration of triblock copolymer in solution plays an important role in the formation of bridged micelles. The higher the concentration, the more micelles may be bridged. So in the present study, the concentration of triblock copolymer in the solution is relatively low. In this paper, the formation of polymeric micelles in an aqueous milieu from PLA/PEG/PLA triblock copolymer is the subject of the studies with the intention of using these polymeric micelles as drug carriers. The nature and stability of self-association formed by PLA/PEG/PLA copolymers in aqueous environments are of fundamental importance as regards their potential drug carrying capacity.

\section{EXPERIMENTAL}

\section{Materials}

$\mathrm{D}, \mathrm{L}$-Lactide was synthesized according to the published procedure. ${ }^{19} \mathrm{D}, \mathrm{L}$-Lactide was recrystallized twice from ethyl acetate prior to use. $\alpha, \omega$-Dihydroxy-PEGs with molecular weights 1000 (PEG 1 K), 2000 (PEG 2 K), and 4000 (PEG $4 \mathrm{~K}$ ) were dried over $\mathrm{P}_{2} \mathrm{O}_{5}$ at $80^{\circ} \mathrm{C}$ under vacuum $(10 \mathrm{mmHg})$. Stannous octoate was purchased from Sigma and used without further purification. All other reagents were analytical grade. 


\section{Synthesis of PLA/PEG/PLA Triblock Copolymers}

PLA/PEG/PLA triblock copolymers were prepared by bulk polymerization. PEG, D,L-lactide, and stannous octoate were introduced into a dried polymerization tube. The mixture was kept under high vacuum at $80^{\circ} \mathrm{C}$ for $2 \mathrm{~h}$ to remove all volatiles. Afterwards the tube was purged with nitrogen three times and sealed under vacuum. The reaction was carried out at $130^{\circ} \mathrm{C}$ for $30 \mathrm{~h}$ without stirring. The product was dissolved in dichloromethane, precipitated in cold ether, and washed with distilled water. The copolymer was dried in vacuum oven at $60^{\circ} \mathrm{C}$ for 3 days.

The compositions of copolymers are determined by ${ }^{1} \mathrm{H}$ NMR (Varian UNITY plus-400 NMR spectrometer, $d$-chloroform) and the molecular weight of PLA/PEG/ PLA copolymers is calculated by the following equation:

$$
M_{n}=(L A / E O \times 144 / 44+1) \times M_{n_{\mathrm{PEG}}}
$$

where $L A / E O$ is the molar ratio of lactide to ethylene oxide in the copolymer determined by ${ }^{1} \mathrm{H}$ NMR, and $M_{n_{\mathrm{PEG}}}$ is the number average molecular weight of PEG. The molecular weight distributions (MWD) of the copolymers were determined by gel permeation chromatography (GPC) on Waters 510 high performance liquid chromatograph using chloroform as eluent solvent. Peaks were detected using a refractive index detector. Polystyrene standards were used to calibrate the system.

\section{Preparation of Micelles in an Aqueous Milieu}

Copolymer micelles were prepared as follows: given amount of triblock copolymer was dissolved in acetone. Then a measured amount of copolymer solution was added dropwise into $10 \mathrm{~mL}$ distilled water under magnetic agitation to form micelles. Acetone was removed under vacuum.

\section{Dynamic Light Scattering Measurements}

Dynamic light scattering (DLS) experiments were performed to measure the hydrodynamic diameter of the micelles. Before measurements, all samples were purified by filtering through a $0.45-\mu \mathrm{m}$ filter (Millipore). Dynamic light scattering measurements were made using an argon ion laser with a wavelength of $514.5 \mathrm{~nm}$ and output power of $10-200 \mathrm{~mW}$. All the DLS was measured at $90^{\circ} \mathrm{C}$ and collected on a Brookhaven BI-9000AT correlator at $25^{\circ} \mathrm{C}$. The measurement for each solution was repeated at least three times.

The translational diffusion coefficient $D$ and the hydrodynamic radius $R_{\mathrm{h}}$ were obtained from the average decay rate $\Gamma$ and Stokes-Einestein formula,

$$
D_{\mathrm{T}}=\Gamma / q^{2}
$$

and

$$
R_{\mathrm{h}}=k T / 6 \pi \eta D_{\mathrm{T}}
$$

where the scattering vector

$$
q=4 \pi n / \lambda \sin (\theta / 2)
$$

in eq $2-4 \theta, \lambda, k, T, \eta$, and $n$ are the scattering angle, the incident wavelength in vacuum, Boltzmann constants, temperature, solvent viscosity, and solvent refractive index, respectively. Using eq $2-4$ the hydrodynamic diameter $2 R_{\mathrm{h}}$ can be calculated. The polydispersity of micelles $\left(\mu / \Gamma^{2}\right.$, where $\mu$ is the second cumulant of the decay function) could be obtained using CONTIN V5.0 software supplied by Brookhaven.

\section{Transmission Electron Microscopy}

Transmission electron microscopy (TEM) was used to observe the morphology of the micelles. Specimens were prepared by dropping the sample solution into copper grid. The grid was held horizontally for $20 \mathrm{~s}$ to allow the molecular aggregates to settle and then at $45^{\circ}$ to allow excess fluid to drain. The grid was returned to the horizontal position and a drop of phosphotungstic acid was added to give a negative stain. The grid was then left to stand for $30 \mathrm{~s}$ before removing excess stain as above. Specimens were air dried before examination using a Philips EM400ST transmission electron microscopy at an accelerating voltage of $80 \mathrm{kV}$.

\section{Measurement of Fluorescence Spectroscopy}

The critical micelle concentrations (CMC) of block copolymers were estimated to prove the potential of micelle formation by the measurement of fluorescence spectroscopy using pyrene as a fluorescence probe. ${ }^{6}$

To study the fluorescence spectroscopy characteristics, PLA/PEG/PLA triblock copolymer solutions were prepared as follows: the block copolymer $(50-100 \mathrm{mg})$ was first dissolved in tetrahydrofuran (ca. $5 \mathrm{~mL}$ ). The polymer solution was added with agitation to $20 \mathrm{~mL}$ doubly distilled water in a $50 \mathrm{~mL}$ volumetric flask. Tetrahydrofuran was evaporated under reduced pressure for $1 \mathrm{~h}$. Finally, this solution was diluted with water to $50.0 \mathrm{~mL}$.

To obtain sample solutions, a known amount of pyrene in acetone was added to each of a series of $10 \mathrm{~mL}$ volumetric flask and the acetone evaporated. The amount was adjusted to give a pyrene concentration in the final solution of $1.2 \times 10^{-7} \mathrm{M}$. A measured amount of copolymer solution was added to each flask, followed by doubly distilled water. The flask were then heated for $3 \mathrm{~h}$ at $65^{\circ} \mathrm{C}$ to equilibrate the pyrene and the micelles and subsequently left to cool overnight at room temperature.

Fluorescence measurements were carried out using a Shimadzu RF-5000 fluorescence spectrometer at an excitation wavelength of $335 \mathrm{~nm}$.

\section{RESULTS AND DISCUSSION}

The polymers examined in the experiments were prepared by ring-opening bulk polymerization of $\mathrm{D}, \mathrm{L}-$ lactide initiated with hydroxy-terminated PEG. Compositions, molecular weights and molecular weight distributions (MWD) of PLA/PEG/PLA triblock copolymers are presented in Table $\mathrm{I}$.

It is well known that amphiphilic block copolymers with suitable hydrophilic/hydrophobic balance can form micellar structures when exposed to a selective solvent. ${ }^{5}$ In the preparation of micelles from PLA/PEG/PLA triblock copolymers, water was employed which is a good solvent for PEG and a poor solvent for PLA. In the present study, a solvent diffusion method was employed to prepare the polymeric micelle; that is, an acetone solution of the block copolymer was exposed 
Table I. Molecular characteristics of PLA/PEG/PLA triblock copolymers

\begin{tabular}{crrrrr}
\hline Sample & $M_{n_{\mathrm{PEG}}}$ & $M_{n_{\mathrm{PLA}}{ }^{\mathrm{a}}}$ & $M_{n_{\mathrm{tota} 1}}$ & $\mathrm{MWD}^{\mathrm{b}}$ & $\mathrm{PEG} / \mathrm{wt} \%$ \\
\hline PLA/PEG2K/PLA-1 & 2000 & 18000 & 38000 & 1.24 & 5.2 \\
PLA/PEG4K/PLA-2 & 4000 & 18000 & 40000 & 1.73 & 10.0 \\
PLA/PEG1K/PLA-3 & 1000 & 8500 & 18000 & 1.29 & 5.6 \\
PLA/PEG1K/PLA-4 & 1000 & 3600 & 8200 & 1.35 & 12.1 \\
PLA/PEG1K/PLA-5 & 1000 & 1850 & 4700 & 1.25 & 21.0
\end{tabular}

${ }^{a}$ Determined by ${ }^{1} \mathrm{H}$ NMR. ${ }^{\mathrm{b}}$ Determined by GPC.

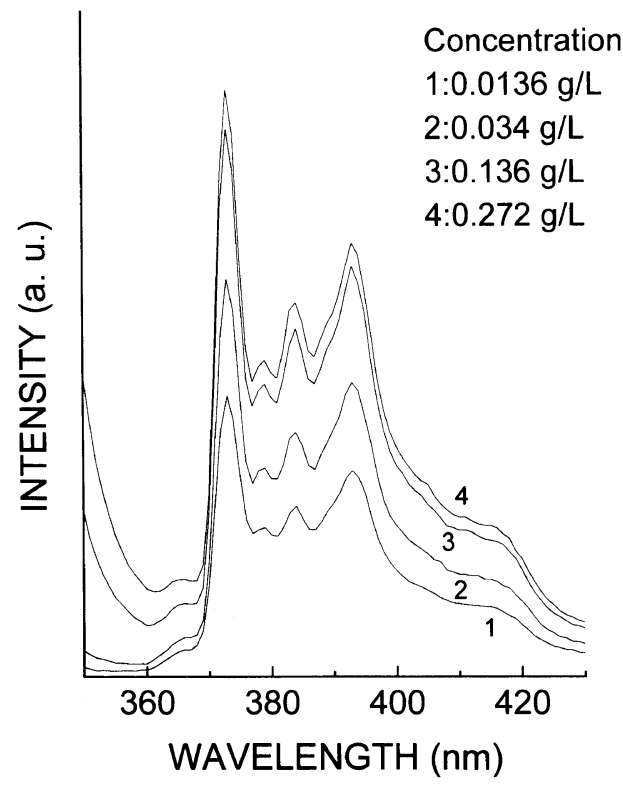

Figure 1. Fluorescence emission spectra of pyrene against concentration of triblock copolymer PLA/PEG2K/PLA-1 in distilled water. Excitation wavelength: $\lambda_{\mathrm{ex}}=335 \mathrm{~nm}$.

to water and then acetone was evaporated at reduced pressure.

\section{FLUORESCENCE PROBE EXPERIMENTS}

$\mathrm{CMC}$ is a measure to describe the physical properties of the micelle and refers to the micelle stability. ${ }^{20}$ The term is actually derived from low molecular weight micelles, but it is also an appropriate measure to characterize the stability of polymeric micelles. Pyrene is a widely used fluorescence probe because it partitions preferably into hydrophobic microdomain and causes changes in the photophysical properties of micelles. ${ }^{21}$ Fluorescence spectra of the PLA/PEG2K/PLA-1 triblock copolymer sample at various concentrations in the presence of $1.2 \times 10^{-7} \mathrm{M}$ pyrene are shown in Figure 1 . The sample is excited at $335 \mathrm{~nm}$. The spectrum is typical of pyrene fluorescence. ${ }^{6}$ The total fluorescence intensity increased with increasing polymer concentrations. Moreover, the vibrational fine structure of pyrene monomer emission spectrum changed upon the formation of polymeric micelle, due to changes in the total polarity, as the transfer of pyrene from a polar environment to a nonpolar one suppresses the allowedness of the symmetry-forbidden $(0,0)$ - the Ham effect. This change is described in terms of the ratio $I_{1} / I_{3}$, the intensities, respectively, of the first and third bands in the pyrene fluorescence spectrum. ${ }^{6}$ The $I_{1} / I_{3}$ ratio is
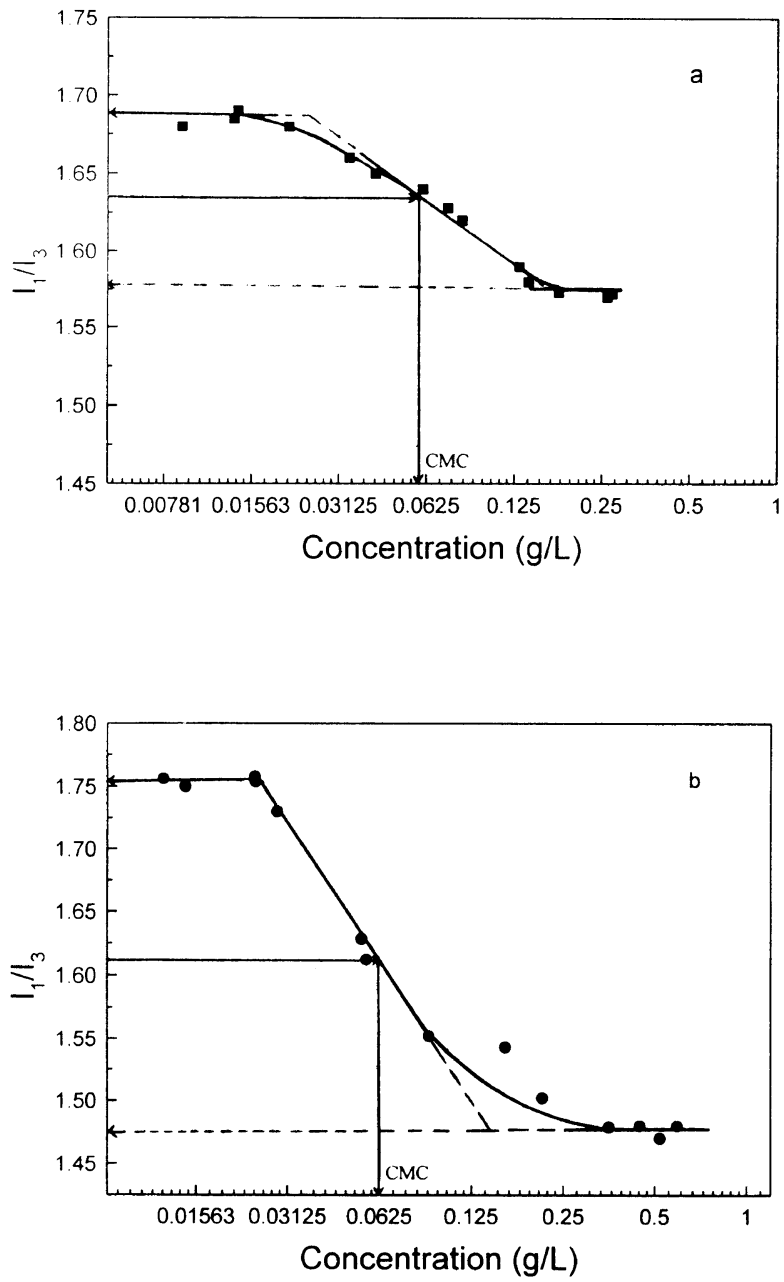

Figure 2. Plot of the intensities $\left(I_{1} / I_{3}\right)$ of the pyrene fluorescence spectrum as a function of triblock copolymer concentrations in distilled water. a, PLA/PEG2K/PLA-1; b, PLA/PEG4K/PLA-2.

very helpful for determining the location of the pyrene probe in the micelles.

A plot of the $I_{1} / I_{3}$ band intensity ratio of pyrene monomer as a function of PLA/PEG2K/PLA-1 (Figure 2a) and PLA/PEG4K/PLA-2 (Figure 2b) triblock copolymer concentrations is shown in Figure 2. Below a certain concentration, $I_{1} / I_{3}$ is essentially constant, and above this concentration, this value decreases with increasing concentration, which indicated partitioning of this hydrophobic probe between the hydrophobic core of micelle and aqueous phase. Pyrene in water shows high value of $I_{1} / I_{3}$, which decreases substantially upon transfer to the less polar micellar domain. The values of the CMC based on the change in the $I_{1} / I_{3}$ band intensity ratio of pyrene were $59 \mathrm{mg} \mathrm{L}^{-1}$ for PLA/PEG2K/PLA-1, and $62.5 \mathrm{mg} \mathrm{L}^{-1}$ for PLA/PEG4K/PLA-2, respectively. Increasing the PEG chain length, the $\mathrm{CMC}$ value does not change very much. This result shows that the PEG chain length has insignificant effects on the CMC values, which agrees well with the result of Mattice. ${ }^{22}$ Mattice et al. studied micellization of symmetric triblock copolymer with insoluble end block using the Monte Carlo technique. Their results indicated that although there is a slight increase in the CMC as the middle block length increase, this variation is relatively small. 

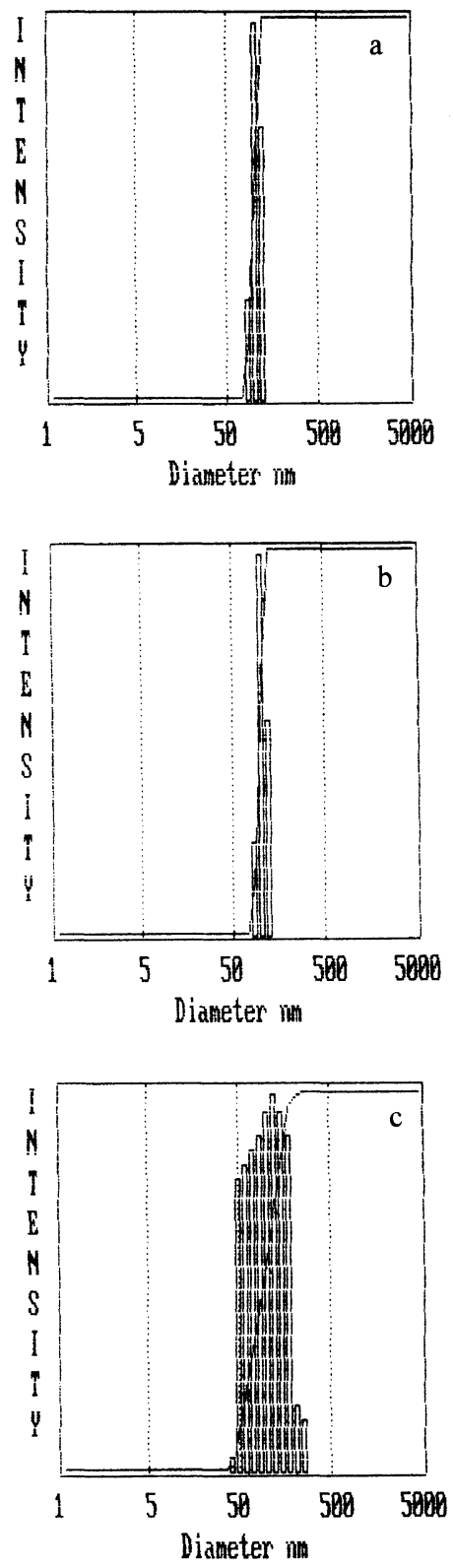

Figure 3. Size distribution of PLA/PEG/PLA micelles determined by dynamic light scattering. Triblock copolymer concentration, $0.25 \mathrm{~g} \mathrm{~L}^{-1}$. a, PLA/PEG1K/PLA-3; b, PLA/PEG1K/PLA-4; c, PLA/PEG1K/ PLA-5.

\section{DYNAMIC LIGHT SCATTERING (DLS) MEASUREMENTS}

The size and size distribution of the obtained micelles were estimated by DLS measurement. Representative data for the distribution of the obtained polymeric micelles are shown in Figure 3. It is seen from Figure 3 that the PLA/PEG/PLA triblock copolymer micelles possess a narrow unimodal distribution in histogram analysis. The micellar size and polydispersity factor determined by a cumulant method are summarized in Table II. The micelle prepared at low concentration by the solvent diffusion method shows low polydispersity. The data in Table II indicated that the diameter of the polymeric micelles was influenced by the molecular weight and the composition of two components of the triblock copolymer. The experimental results in Figure 3 and Table II notably show that the hydrodynamic 848
Table II. Size and polydispersity factor of PLA/PEG/PLA micelles prepared in aqueous milieu

\begin{tabular}{crrrc}
\hline Sample & $L_{\mathrm{PEG}}{ }^{\mathrm{a}}$ & $L_{\mathrm{PLA}}{ }^{\mathrm{b}}$ & $D / \mathrm{nm}$ & Polydispersity \\
\cline { 4 - 5 } & & & & $\mu / \Gamma^{2}$ \\
\hline PLA/PEG2K/PLA-1 & 45 & 253 & 144.0 & 0.13 \\
PLA/PEG4K/PLA-2 & 91 & 250 & 129.5 & 0.11 \\
PLA/PEG1K/PLA-3 & 23 & 117 & 134.9 & 0.06 \\
PLA/PEG1K/PLA-4 & 23 & 50 & 123.5 & 0.08 \\
PLA/PEG1K/PLA-5 & 23 & 26 & 120.4 & 0.14
\end{tabular}

${ }^{a} L_{\text {PEG }}$, average segment length of PEG block. ${ }^{\mathrm{b}} L_{\mathrm{PLA}}$, average segment length of PLA block.

radius of micelles is dependent on the PLA chain length. When the molecular weights of PLA block are 8424 , 3600 , and 1872 , the hydrodynamic radius of micelles are $134.9,123.5$, and 120.4 , respectively, i.e., the longer the PLA chain length, the larger the micelle size. Balsara et al. ${ }^{23}$ pointed out that for BAB triblock copolymer in the solvent that preferentially dissolves the A block, the increase in the molecular weight of the poorly solvated block tends to favor micelle formation. Both the number of block copolymer chains in a micelle and average radius of the micelles increase with the increasing of molecular weight of B block.

\section{MORPHOLOGY OF POLYMERIC MICELLES}

The morphology of the micelles was investigated by transmission electron microscopy technique. Figures $4 \mathrm{a}$ and $4 \mathrm{~b}$ show transmission electron micrographs of PLA/PEG2K/PLA-1 and PLA/PEG4K/PLA-2 copolymer micelles, respectively. The two pictures clearly indicate the presence of micelles as shown by light spherical entities surrounded by the dark staining. The size of micelles estimated from TEM photographs was approximately in the range of 19 to $65 \mathrm{~nm}$ for PLA/ PEG2K/PLA-1, and 15 to $57 \mathrm{~nm}$ for PLA/PEG4K/ PLA-2, respectively. The size of micelles obtained from TEM photographs is much smaller than that measured by DLS. The difference should be due to the collapse of hydrophilic corona of micelles during the preparation of TEM specimens.

\section{IN VITRO DEGRADATION BEHAVIOR}

PLA/PEG/PLA triblock copolymers contain two types of sites where hydrolysis could occur, namely ester bonds within the PLA blocks and ester bonds linking PEG block to PLA blocks. The hydrolysis at the two sites proceeded with comparable rates. ${ }^{16}$ So it is interesting to investigate the influence of copolymer hydrolysis upon the size and morphology of micelles. The in vitro hydrolytic behavior of polymeric micelles of PLA/ PEG2K/PLA-1 block copolymer was studied in phosphate buffer solution ( $\mathrm{PBS}, \mathrm{pH}=7.4$ ) at room temperature. The GPC traces of PLA/PEG2K/PLA-1 copolymer before hydrolysis and after hydrolysis are given in Figure 5. It can be found that the average molecular weight decreases after hydrolysis. The molecular weight distribution of copolymer is unimodal (curve a) prior to hydrolysis, however, after 13 days of hydrolysis, the 

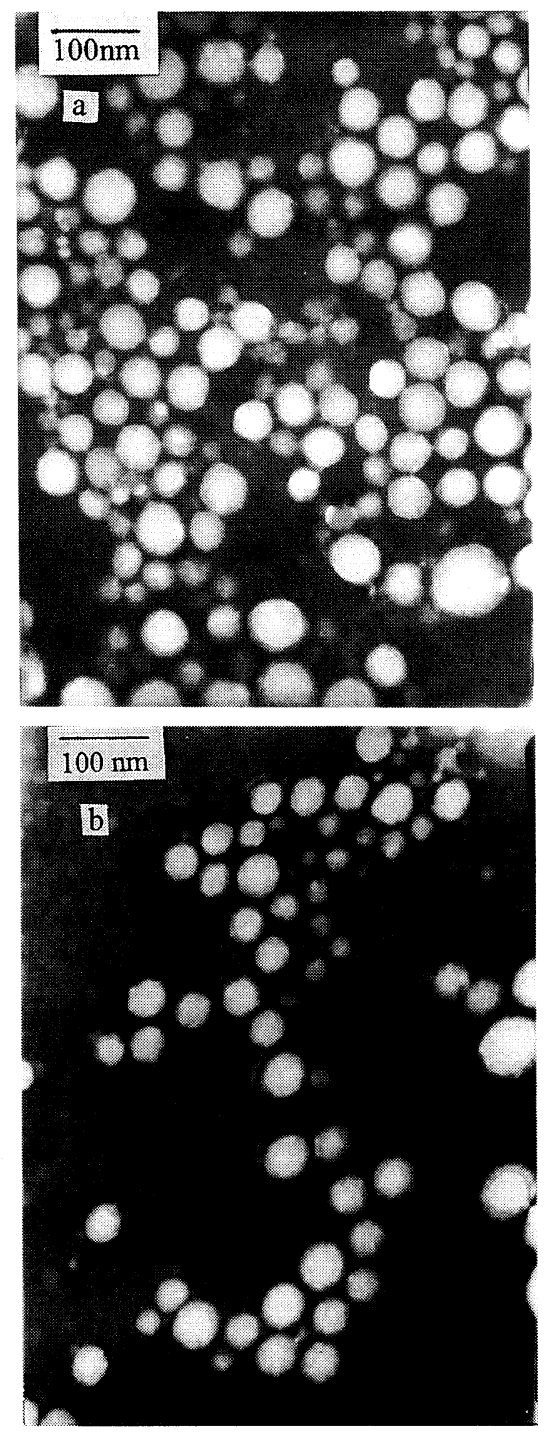

Figure 4. Transmission electron microscopy photographs of PLA PEG/PLA micelles. a, PLA/PEG2K/PLA-1; b, PLA/PEG4K/PLA-2.

molecular weight distribution changes to bimodal and shifts towards the low molecular weight (curve b), which is attributed to the degradation of the copolymer. This result agrees well with what occurred in the hydrolysis of PEG/PLLA at $\mathrm{pH} 7.0$ reported by $\mathrm{Hu}$ et al. ${ }^{18}$

The size distributions of micelles before hydrolysis and after 13 days of hydrolysis were also studied by DLS (Figure 6). The micelles have a narrow unimodal distribution before hydrolysis (Figure 6a). However, after 13 days of hydrolysis, the micelles show a broad biomodal distribution (Figure 6b). In addition, it is seen from the histogram analysis that the change of micellar size shows two opposite tendencies after 13 days of hydrolysis in phosphate buffer solution (PBS). Compared with the micelles before hydrolysis, one part of the micelles became smaller, while, the other part became larger. The decrease in micellar size is attributed to the degradation of PLA block in the copolymer, however the larger micellar size may be due to the secondary aggregation of micelles. However, the details about the secondary aggregates are still unclear. There exist several possible structures after secondary aggregation, such as single large core-shell micelles, onionlike particles with

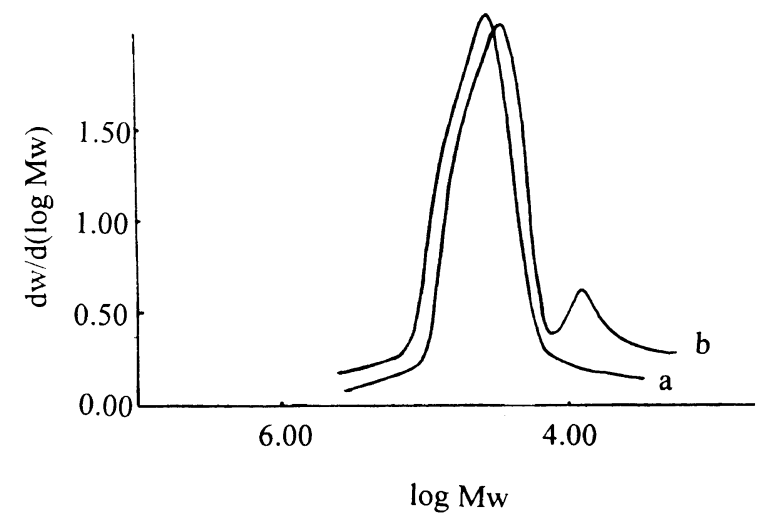

Figure 5. GPC traces of the original (a) and degraded (b) PLA/PEG2K/PLA-1 copolymer.
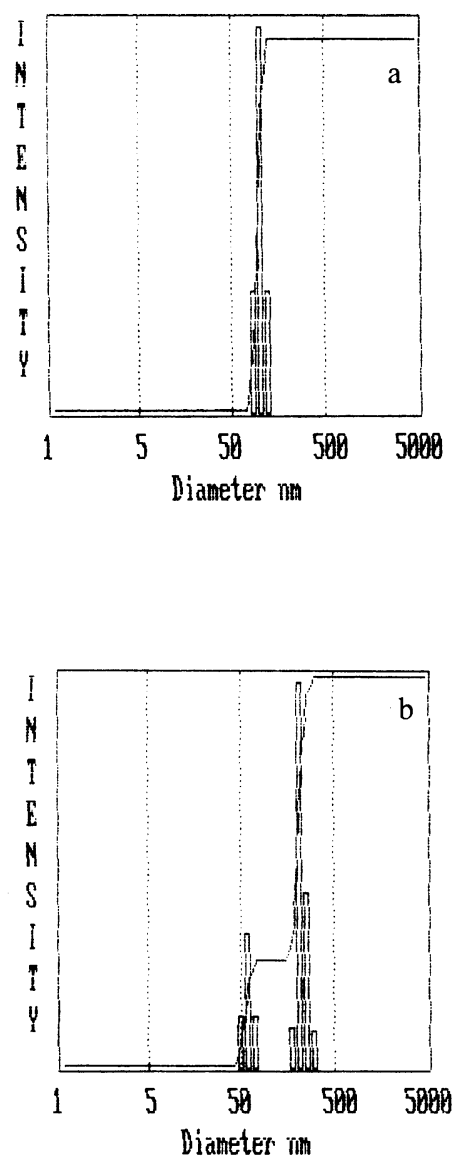

Figure 6. The size distributions of micelles before hydrolysis (a) and after hydrolysis in phosphate buffer solution for 13 days (b). Concentration, $0.25 \mathrm{~g} \mathrm{~L}^{-1}$.

alternating concentric layers of solvated and undissolved blocks, loose clusters of small regular micelles, and further association of micelles by hydrophobic interactions or by the van der Waals interactions between exposed cores as the same principles for formation of micelles and incomplete dissociation of the bulk polymer, etc. $^{24-26}$

In TEM pictures it can also observe some kind of morphological deformation of micelles accompanying the in vitro hydrolytic degradation of copolymer (Figure 7). The micelles before hydrolysis appear spherical in shape (Figure 4). After 13 days of hydrolysis, the mi- 


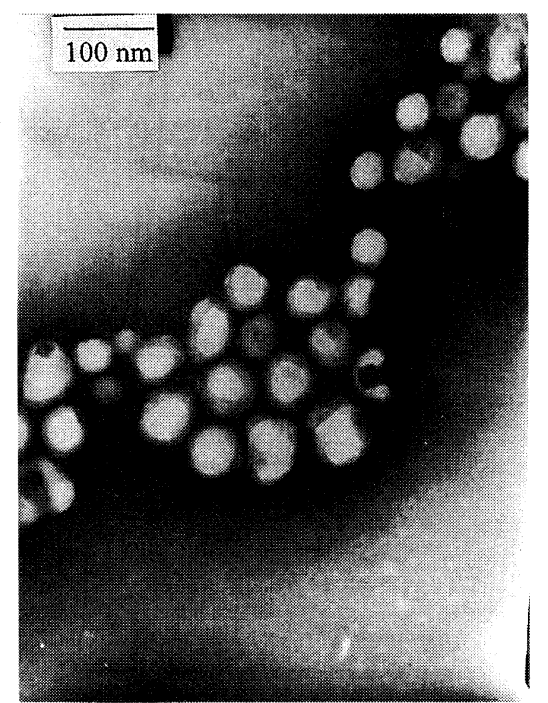

Figure 7. Transmission electron microscopy photograph of micelle after 13 days of hydrolysis in phosphate buffer solution.

celles have some deformation and no longer appear spherical shape.

The above experimental data including GPC, DLS, and TEM results show that the copolymer undergoes degradation after 13 days of hydrolysis in PBS at $\mathrm{pH}$ 7.4. The hydrolysis of the copolymer exerts evident influence upon the size and morphology of the micelles.

\section{CONCLUSION}

The amphiphilic triblock copolymers PLA/PEG/PLA formed micelles in aqueous milieu. Fluorescence spectroscopy measurements suggested that PLA/PEG/PLA triblock copolymers were assembled to form coreshell type polymeric micelles in water and $\mathrm{CMC}$ values changed relatively small with increasing PEG chain length. Micelles were spherically shaped and had narrow size distributions. The size of micelles increased with increasing PLA chain length. After 13 days of

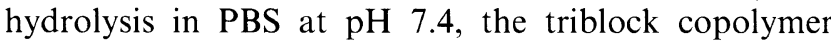
proceeds degradation, and the degradation has some influences upon the size distribution and morphology of the micelles. These biodegradable polyether-polyester triblock copolymer micelles may have potential for parenteral drug delivery systems.
Acknowledgment. The authors thank Dr. Hanying Zhao for his beneficial discussions. This project is supported by China Postdoctoral Science Foundation and Doctoral Foundation of Chinese University.

\section{REFERENCES}

1. R. Gref, Y. Minamitake, M. Peracchia, Y. Trubetskoy, V. Torchilin, and R. Langer, Science, 263, 1600 (1994).

2. A. Harada and K. Kataoka, Macromolecules, 31, 288 (1998).

3. A. Harada and K. Kataoka, Macromolecules, 28, 5294 (1995).

4. J. W. Nah, Y. I. Jeong, and C. S. Cho, J. Polym. Sci., Part B, Polym. Phys., 36, 415 (1998).

5. Z. Tuzar and P. Kratochvil, Adv. Colloid Interface Sci., 6, 201 (1976).

6. M. Wilhelm, C. L. Zhao, Y. Wang, R. Xu, and M. A. Winnik, Macromolecules, 24, 1033 (1991).

7. I. Astafieva, X. F. Zhang, and A. Eisenberg, Macromolecules, 26, 7339 (1993)

8. A. Qin, M. Tian, C. Ramireddy, S. E. Webber, P. Munk, and Z. Tuzar, Macromolecules, 27, 120 (1994).

9. H. W. Daly and D. Poche, Tetrahedron Lett., 29, 5859 (1988).

10. M. Yokoyama, M. Miyauchi, N. Yamada, T. Okano, Y. Sakurai, K. Kataoka, and S. Inoue, J. Controlled Release, 11, 269 (1990).

11. G. Kwon, S. Suwa, M. Yokoyama, T. Okano, Y. Sakurai, and K. Kataoka, J. Controlled Release, 29, 17 (1994).

12. S. A. Hagan, A. G. A. Coombes, M. C. Garnett, S. E. Dunn, M. C. Davies, L. Illum, S. S. Davis, and S. E. Harding, Langmuir, 12. 2153 (1996).

13. D. Bazile, C. Prud'homme, M. T. Bassoulet, M. Marlard, G. Spenlehauer, and M. Veillard, J. Pharm. Sci., 84, 493 (1995).

14. D. A. Herold, K. Keil, and D. E. Bruns, Biochem. Pharmacol., 38, 73 (1989).

15. A. W. Richter and E. Akerblom, Int. Arch. Allergy Appl. Immunol., 70, 124 (1983).

16. I. Rashkov, N. Manolova, S. M. Li, J. L. Espartero, and M. Vert, Macromolecules, 29, 50 (1996).

17. S. M. Li, I. Rashkov, J. L. Espartero, N. Manolova, and M. Vert, Macromolecules, 29, 57 (1996).

18. D. S. Hu and H. J. Liu, J. Appl. Polym. Sci., 51, 473 (1994).

19. D. K. Gilding and A. M. Reed, Polymer, 20, 1459 (1979).

20. G. S. Kwon, M. Naito, M. Yokoyama, T. Okano, Y. Sakurai, and K. Kataoka, Langmuir, 9, 945 (1993).

21. K. C. Dowling and J. K. Thomas, Macromolecules, 23, 1059 (1990)

22. M. N. Misra and W. L. Mattice, Macromolecules, 28, 1444 (1995).

23. N. P. Balsara, M. Tirrell, and T. P. Lodge, Macromolecules, 24, 1975 (1991)

24. T. N. Kahn, R. H. Mobbs, C. Price, J. R. Quintana, and R. B. Stubbersfield, Eur. Polym. J., 23, 191 (1987).

25. R. Xu, M. A. Winnik, F. R. Hallett, G. Riess, and M. O. Croucher, Macromolecules, 24, 87 (1991).

26. Z. Gao and A. Eisenberg, Macromolecules, 26, 7353 (1993). 